\title{
NUMERICAL ERROR ANALYSIS FOR CONFIGURABLE CELL SEGMENTATION PROBLEM
}

\section{Nataliya BILOUS ${ }^{1 *}$, Oleg HRAMM${ }^{1}$, Iryna AHEKIAN ${ }^{1}$, Abed Thamer KHUDHAIR ${ }^{2}$, Ludmila ILLYASHENKO ${ }^{3}$, Alexander NERUKH ${ }^{4}$}

\author{
${ }^{1}$ Department of Software Engineering, Faculty of Computer Sciences, Kharkiv National University of Radio Electronics, \\ Kharkiv, Ukraine \\ 2 Department of Informatics, Kuliyyah Al-Maarif University College, Anbar, Irag \\ ${ }^{3}$ Outsourcing Scientific Training Pproduction Center, Kharkiv National University of Radio Electronics, Kharkiv, Ukraine \\ ${ }^{4}$ Department of Higher Mathematics, Faculty of Information and Analytical Technologies and Managment, Kharkiv, \\ Ukraine
}

\begin{abstract}
The current intense interest in gold nanoparticles is due to their Surface Plasmon Resonances (SPR) that depend strongly on the shape and size of the nanoparticles. As the SPR wavelength and resonantly enhanced absorption and scattering properties also depend on the dielectric medium in which gold nanoparticles are embedded, and also depend on the way of their clustering, they are useful to design novel nanodevices, in particular when it is based on ideas taken from nature. With purpose to select the most promising configurations for novel nanodevice design in this work the method of cell recognition and evaluation of its efficiency is proposed. Exist different methods to produce microscopic images, they can be obtained for different types of cells in different environments. Due to this fact, the recognition algorithms are needed. All methods have their advantages and disadvantages and may work well only under certain conditions. Therefore, it is useful for each specific task to implement a separate algorithm that will be effective for the existing set of images, and take into account the peculiarities of these images. The task of this work is not only to develop flexible and customizable algorithm, that can be configured to segment cells on different types of images, but also provide numerical error analysis corresponding to each step of algorithm. As a result, a solution is developed, that has many customizable parameters to optimize the result for a specific data set and specific accuracy. In addition, this it is resistant to a lot of noise and artifacts, that can occur on images, such as uneven background, small debris, loss of focus when shooting. Numerical error analysis allows getting form of cell segmentation more precisely to be reproduced for novel nanostructured device design.
\end{abstract}

Keywords: Cells recognition, Segmentation, Watershed; Hough transform, Plasmonic nanoparticles, Error estimation

\section{INTRODUCTION}

Man is never satisfied with stagnation, continuing to invent new better, cheaper, faster, lighter and smaller devices; however it is difficult to invent something completely new, because most new proposals already exist somewhere. With the benefits of millions of years of natural selection, it is no surprise that when it comes to fitting form to function, nature engineered the right structure for the right job. Nature provides a wide range of complex solutions so that one may pick natural templates, which are almost perfectly suited for the engineers to be performed, in particular when fragment of an image with cells is investigated carefully, bringing at the same time new discoveries in nanosience as well as nanoengeneering. It is not possible not to mention the most famous historical fact when Joseph Paxton, as Head Gardener to the Duke of Devonshire, used the mechanical stability of lily leaves as the catalyst for his designs of conservatories and glasshouses. He went on to design the Crystal Palace of the Great Exhibition 1851 built of glass and cast iron using multiple arrays of radiating ribs connected with crossribs.

Scientists have taken many innovative ideas from the biological world, e.g. burs on a dog's coat led to the invention of Velcro, investigations into iridescence on butterflies and beetles led to brighter screens

*Corresponding Author: nataliya.bilous@nure.ua

Received:14.10.2019 Published: 16.12.2019 
for mobile telephone handsets, the finger-like primary feathers of raptors led to developing wings that change shape aloft to reduce drag and increase fuel efficiency.

It is thus no surprise that in the development of advanced nanoscale devices man once again turns to nature! To increase the brightness of electroluminescent nanodevices the eyes of nocturnal animals with high utilization efficiency of light were considered [1]. The surface nanotextures of block copolymers that are found in many products, including shoe soles, adhesive tapes and automotive interiors, mimic those found in nature, such as the tiny light-trapping posts that make moth eyes dark to help the insects avoid detection by predators and the waxy cones that keep cicada wings clean [2].

The investigation of the properties for several natural materials give ideas to researchers to invent new nanostructured artificial materials, attempting to reproduce Nature's achievements. Water-pinning nanostructures [3], high-performing nanocomposite materials with ideas taken after consideration of mussels and seashells [4], a method for tissue regeneration [5], even a bio-inspired cement [6] already were invented. A set of robust bio-inspired nanocomposites including other functions is considered in [7] with application to nanogenerators, electrical conductors, supercapacitors, actuators, strain sensors and fire retardancy.

Most biological structures like cells, bacteria and viruses come in a variety of shapes. Among others are tubes, rods, and squashed spheres, or ellipsoids. Producing the shape inspired by nature, plastic nanoparticles, improved cancer drug delivery. This is due to the fact that plastic nanoparticles with natural shape enter tumour cells more easily [8].

In this work, the shape of cells and their arrangement were investigated more precisely since artificial nanoparticles and nanostructures, which repeat those taken from nature, hold promise for design of novel nanodevices.

The principles of constructing the computer vision system imply the presence of few levels, such as preprocessing, filtration, segmentation and classification. First of all, the pre-processing phase, changes the properties of the image to optimize the result in further processing steps. Second, the filtering phase, removes areas, that do not contain important information and complicate the segmentation process. Third, the segmentation phase separates the elements and their limits, which ultimately improves the quality and accuracy of the diagnosis [9]. Each of these phases has its own set of algorithms. While the existing algorithms for pre-processing and filtering phases allow qualitatively meeting all the requirements for these levels, the segmentation phase still pose challenging problems when solving the task for specific data with specific accuracy. So, traditionally used image segmentation algorithms, based on the principle of proximity of object points by color (brightness) and do not take into account the intersections of objects, exclude the possibility of further classification with an acceptable [10]. Also, cell structures may look completely different, have a transparent, translucent or opaque structure. Other problems arise due to the boundaries. In addition, images can have a lot of noise and other due to objects that should be excluded from processing. The most important is that all these properties manifest themselves differently on different data sets. So, it is urgently needed to develop algorithms for specific classes of images with increased effectiveness [11]. The purpose of the current work is to obtain a solution, that will allow the algorithm to be implemented in such a way, so that it is applicable to different data sets and provides needed result. Good algorithm was proposed in the paper [12], using a watershed algorithm for cell segmentation and Hough transform for clustering intersecting cells. The goal is achieved by giving ability to configure the algorithm for the properties of a specific data set. So the novelty of this work lies in the versatility and the possibility of optimization for a different range of segmentation tasks. 
This paper describes the implementation of the modified method [12], so that we can parameterize this algorithm for the most efficient use of different data sets with the ability to optimize the result by configuring this algorithm for a specific data set. In addition numerical error analysis is provided for each step of implemented algorithms. That leads us to the result with over performed efficiency, best possible accuracy, minimizing sufficiently the solution time and the computer resources. The experiments were performed with open source code using the OpenCV framework for Windows OS.

\section{METHOD AND MATERIALS}

Among the many different materials currently in use for nanotechnology, metal nanoparticles are extremely attractive. In most of works for plasmonic applications two types of plasmonic metal, gold and silver, are considered. Although the use and importance of gold in the world is jewellery and finance is well known, it is not surprising that it is found in many less glamorous applications. This is because one of the gold's main advantages for many applications is that it does not oxidize. Therefore in medicine a large community of researchers is investigating the use of gold nanoparticles for cancer treatment. Such drugfree approaches involve heating the gold nanoparticles with radiation so that they fry the tumor. Silver also demonstrated to be good for nanotechnology and this looks set to continue. While both noble metals have survived the ravages of war and pillage, nature, neglect, and numerous experiments, for today's nanophotonic a miracle of silver nanoparticles is not their improbably longevity, but rather their impermanence. For both silver and gold the relatively intense valence-loss spectral region can be interpreted using the classical response theory based on the complex, wavelengthdependent dielectric function $\varepsilon(\lambda)$ for each plasmonic nanoparticle. The spectra of dielectric function of gold and silver are shown in Figure 1. The differences in the properties of two metals are significant, concerning especially the imaginary parts of their dielectric functions at wavelengths shorter that 580 $\mathrm{nm}$. In this work, we provide investigation of gold nanoparticles (Au NPs) with shapes, which are similar to those of cell, obtained with image processing algorithm.

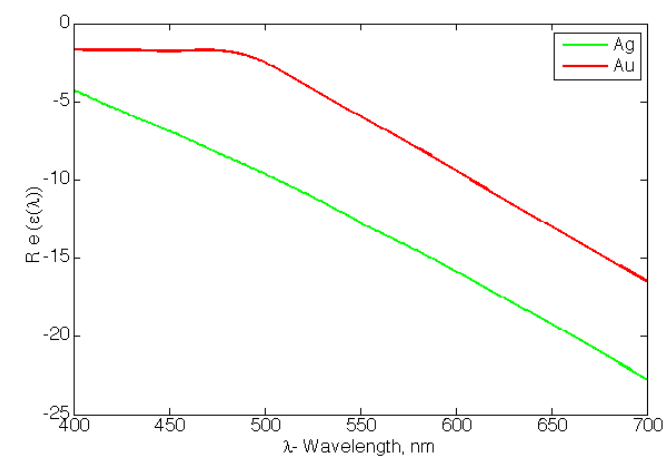

(a)

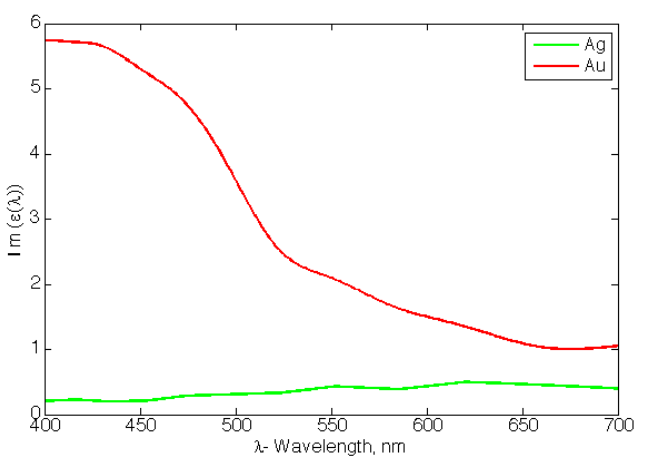

(b)

Figure 1. Spectrum of the dielectric functions of gold and silver: (a) real part; (b) imaginary part

Gold nanoparticles demonstrated to be useful for various applications due to their surface plasmon resonances (SPR). They result in strongly enhanced absorption and scattering of incident light, that depends on shape of size of nanoparticle. When cluster of gold nanoparticles is arranged, the SPR wavelength and resonantly enhanced absorption and scattering properties of cluster depend on number of particles, and their arrangement. To pick natural template for novel nanostructure design, based on cluster of $\mathrm{Au} \mathrm{NPs}$, we first examine optical microscopy image to get full infomation about cell arrangement. 
To do so, our recently developed algorithm for processing fluorescence and optical microscopy images of cell populations is used. It has many configurable parameters, so that our algorithm can be optimized for concrete properties of processed image. The structure of the algorithm is presented in Figure 2.

As an example of the operation of the algorithm, the process of segmentation of living cells in an image is considered.

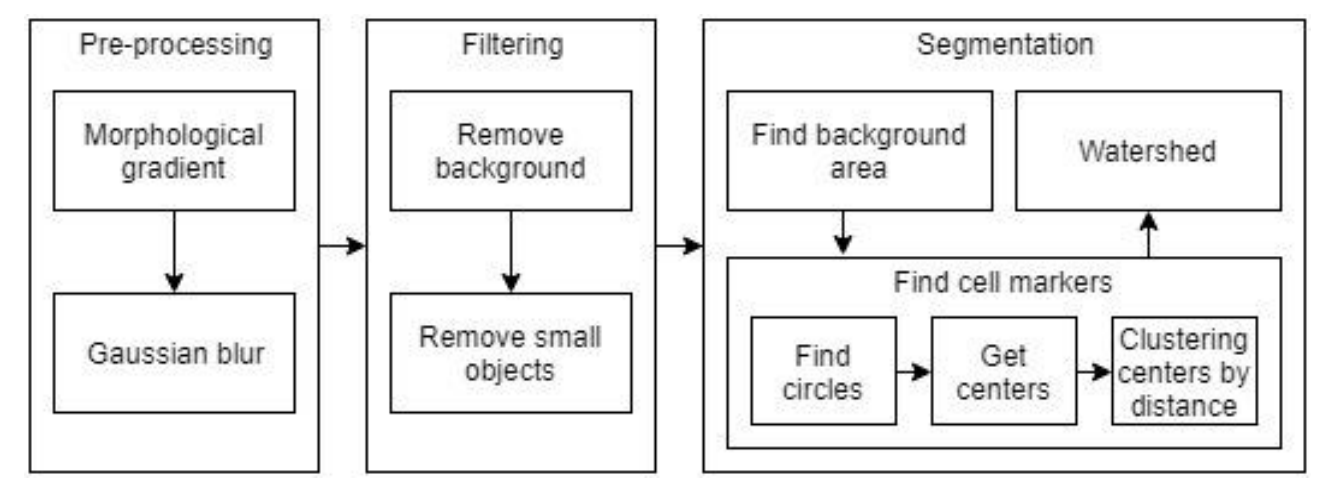

Figure 2. The structure of algorithm.

The image has foreign objects that should not be involved in the segmentation process, and the image also has different brightness in different parts of the background.

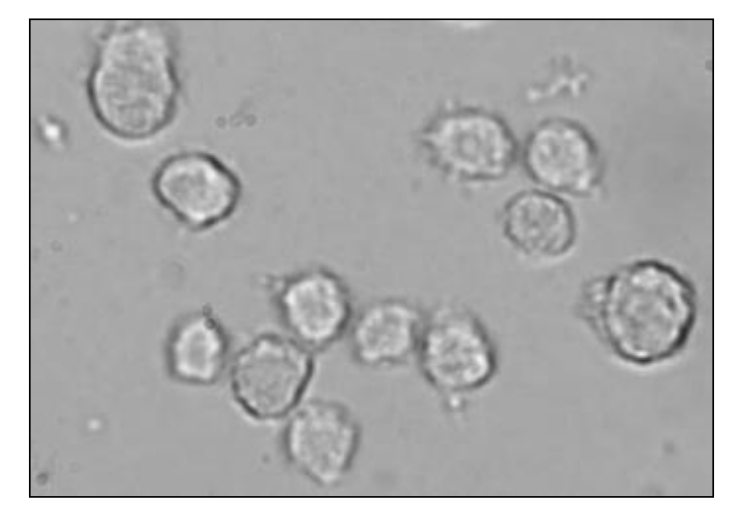

Figure 3. Original image

Then, cells will be segmented for this image using the given algorithm.

\subsection{Pre-processing}

On pre-processing stage, the input image is converted to another image with a different properties. Therefore, the original properties of this image are changed, so that it helps to carry out more effectively the process of filtering and segmentation.

\subsubsection{Correction of background irregularities}

When a lot of images are obtained, and all of them are analysed, it may be seen, that they have different brightness of background areas, which complicates the task of removing it. Morphology transformation is applied to smooth the background. This allows, to remove the dependence of the image on the heterogeneity of the background and leave only the boundaries of the contours [13]. The classical morphological gradient operator for grey scale images is the difference between a dilation and an 
erosion. This is an image in which each pixel value indicates the intensity of the contrast in close proximity to this pixel [14]. As a result, an image is obtained with a uniform level of background brightness for further processing.

\subsubsection{Reducing excess clarity}

Reducing the clarity is necessary to remove unnecessary details. The image is processed using Gaussian blur. Thus, the image is spared from slight unevenness and noise and only the rounded contour of cells remains without image detail. This will make it possible to conduct more effectively the process of locating circles at the segmentation stage, because the search will only produce on the contours and the result will not be affected by noise.

\subsection{Filtering}

Interesting approach was analysed that is based on the method of multi-start optimization with wavelet transformation (MOWT) [15]. This method is used for the filters coefficients tuning because it is less sensitive to starting points of search. But at the filtering stage, all unnecessary data from the image are deleted to get an image containing only the cell area. This is necessary to prepare the image for the next stage - segmentation, because the results of segmentation will be obtained only on the basis of cell data, without the influence of other factors.

\subsubsection{Background removal}

Removing the background using threshold filtering allows to obtain object outlines, separate from the background of the image.

\subsubsection{Filtering small objects}

To filter small objects that are not cells, all continuous groups of pixels, which have a smaller set of pixels than declared in parameters are deleted. The result of filtering steps is presented in Figure 4. This is the original image, for that the morphological gradient was processed, the background removed and small objects are deleted.

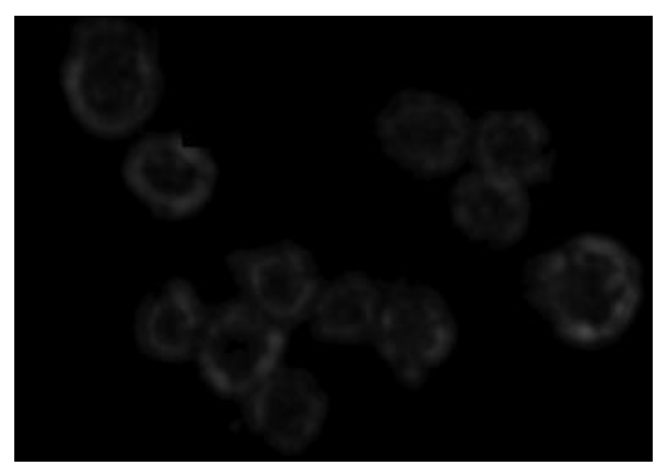

Figure 4. Filtering result

\subsection{Segmentation}

Segmentation of images is one of the most important image processing technologies for biological images. Its goal is to distribute the input image to the regions [13]. The increase in the reliability of the 
results of the segmentation of histological objects is achieved due to the determination of the specific properties of individual objects, it is of an individual nature and cannot be used for the analysis of histological images as a whole [16]. For segmentation, the watershed algorithm is used. For this, background area and cell markers are a set of points, each of which will correspond to one cell must be found.

\subsubsection{Finding background area}

To create a mask from background region, the thresholding binarization algorithm is applied, the result of which is shown in Figure 5 [17]. White marks an area that is certainly the background of the image and does not contain objects.

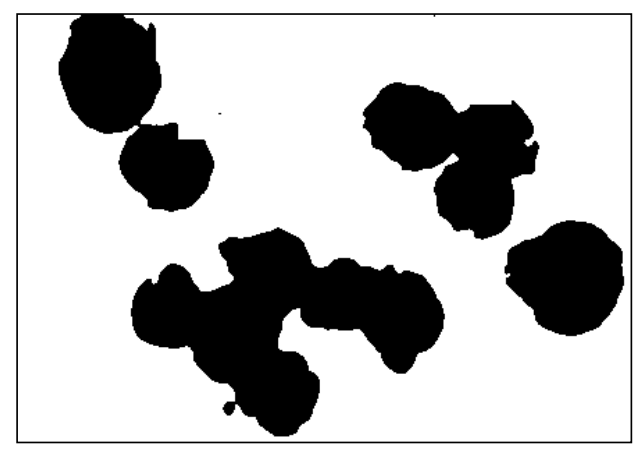

Figure 5. Segments on original image

\subsubsection{Finding cell markers}

The algorithm for finding cell markers contains several steps. The first step is finding circles, that is determination of circles is performed by Hough gradient method [18]. The second step is detection of circle centers, that is done in order to isolate cell markers, while only the centers of the found circles should be left. The third step is clustering of centers by Euclidean distance.

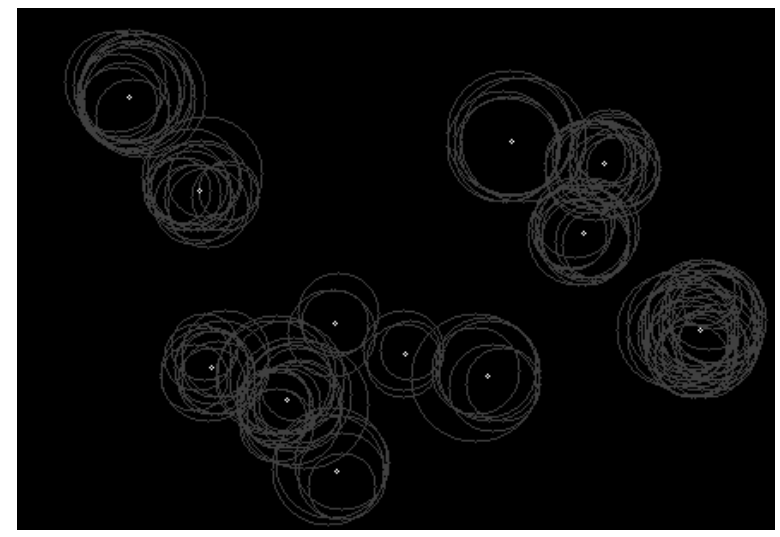

Figure 6. Result of finding circles and clustering

As a result, an image was obtained with cell markers, which can later be used in the watershed algorithm. The result of the marker search is shown in Figure 6. 


\subsubsection{Accepting watershed algorithm}

Applying the previous stages of image processing, an image with cell markers is obtained, and a mask with a region is filled with cells. Having this data the watershed algorithm can be applied. As a result, an image is built, that is presented in Figure 7.

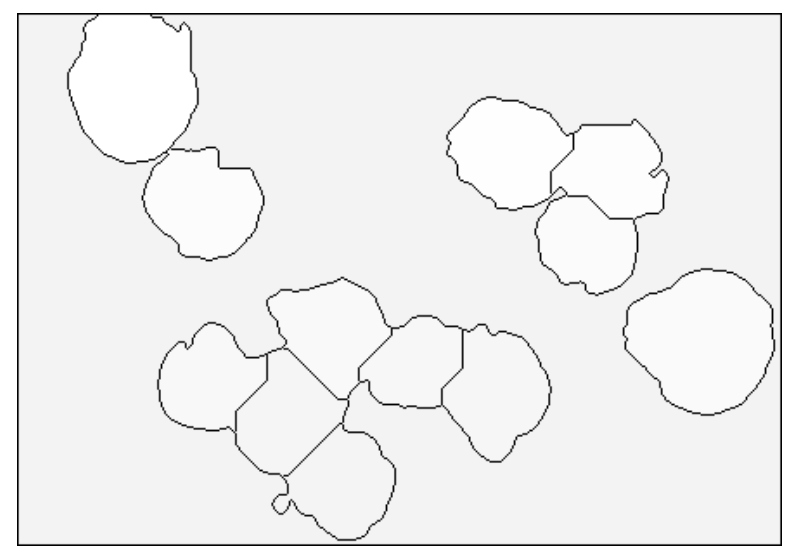

Figure 7. Watershed algorithm result

This is a set of segments, where each segment corresponds to one cell. The resulting segments are used on the source image, presented in Figure 8.

As a result, an image with a set of segments is obtained, where each segment corresponds to one cell. This will allow further implementation of algorithms for analyzing the parameters of cells and their states for each of the segments.

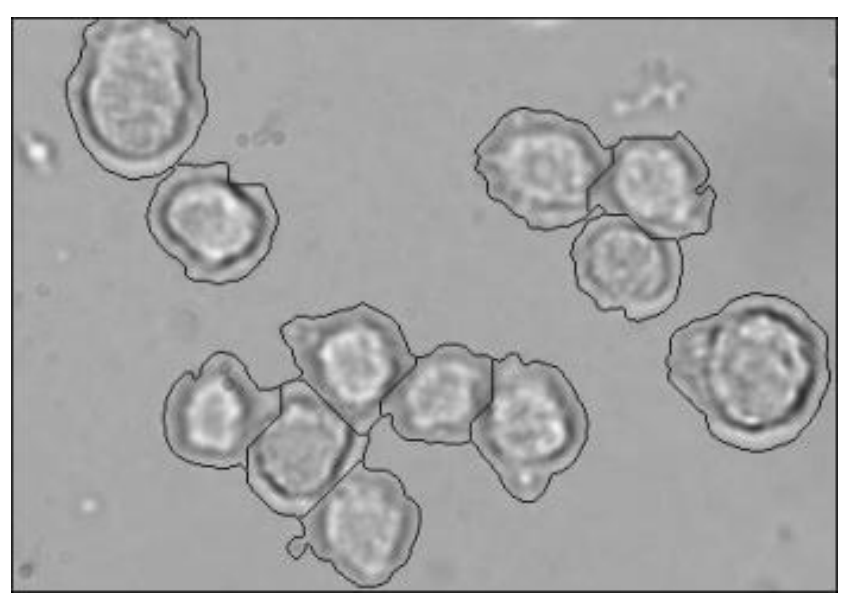

Figure 8. Segments on original image

\subsection{Configured parameters}

In this section we give information about obtained configured parameters. The algorithm has several configurable parameters. This allows to customize the algorithm for a particular data set, which will give opportunity to raise the efficiency when processing it. The available parameters are described below [19]. 
1. Blur level. This parameter changes the intensity of the reduction in the sharpness of the image. The higher the level, the more effective the noise will be eliminated. At excessively high levels, the contours of the cells can be too blurred. If the level of intensity is insufficient, the remaining noise will negatively affect the finding of circles during the search for markers. Effective is the value at which the overall image noise is reduced, but the contours of the cells remain. If the image does not have background noise, the parameter value must be 0 .

2. Background brightness threshold. By means of this parameter gets the brightness value for which all areas with a value below the parameter are considered as the background image. All pixels with a brightness value below the specified parameter will be deleted. At excessively high level the background may be classified as areas that are cells. If the level is insufficient, parts of the background may not be removed and later they will enter the cell segment. Effective is the value at which the largest amount of background and the smallest number of areas with cells will be deleted.

3. External minimum size. By means of this parameter gets the minimum size of objects that will be processed. All objects smaller than the specified size will be deleted from the image. At excessively high level, the areas that are actually engaged in the cell may be removed from the image. If the level is insufficient, the image may contain objects that are not cells and will be defined as separate segments. Effective is the value at which all unnecessary objects will be deleted, but all the areas with the cells will remain unchanged. If the image contains only cells and does not contain extraneous objects to be deleted, the value of the parameter must be 0 .

4. Internal minimum size. Areas that are highlighted as a background and have a size smaller than the specified parameter will be assigned to the area with the cells. At an excessively high value, small areas of the background should be attached to the cell area, which are surrounded by cells. At insufficient level, the middle of some areas with cells can be interpreted as a background and the contour of a segment can be determined incorrectly. Effective is the value at which the contour of the regions with the cells will be as reliable as possible. If all the internal cells areas have not been deleted during the background removal process and the background area is determined correctly, the parameter value must be 0 .

5. Sensitivity of circles detection. This parameter determines how strong the contour of the circle should be and using for adjusting the sensitivity of the definition of circles, during the search for markers. With an excessively high value of the parameter, the actual contour of the cell should not be defined as a circle. With insufficient level also increasing your probability of false circles which will make it difficult to further the process of clustering. The value at which the contours found will coincide with the contours of the cells will be effective.

6. Completeness of circles. This parameter determines how many points of the contour you need to find to define the contour as a circle. The effect is the same as when changing the previous parameter.

7. Minimum circle radius. Parameter determines the minimum size of the circle that could be defined and corresponds to the minimum size of the cell image.

8. Maximum circle radius. This parameter determines the maximum size of the circle that could be defined and corresponds to the maximum size of the image cell.

9. Minimum distance between clusters. This parameter corresponds to the distance between the centers of circles and show when they will belong to one cluster. With excessively high value of the parameter, the points belonging to the regions of different cells should be united into one cluster. With an insufficient parameter, the points belonging to the region of one cell could be divided into different clusters. Effective is the parameter when each cluster will contain one cell. 


\section{RESULTS}

To evaluate the efficiency, a set of images was processed [19, 20,21] with optimized parameters to it determining as the number of cells in the image. The general results are found by processing a sample of near 700 images with a different number of objects and a different probability of intersection with an average error is $2.696 \%$.

\subsection{Dependence on the probability of intersection}

The results of processing, depending on the probability of intersection, were found by processing 300 objects, and have different probability of crossing cells. The results are shown in Figure 9.

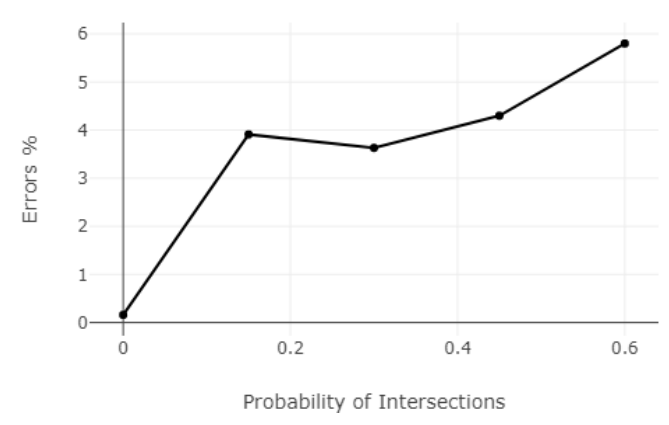

Figure 9. Dependence of probability of intersection to the error

The algorithm without the probability of crossing the cells has the error around $0.16 \%$. But it has a clear correlation between the increase in the probability of crossing cells and the increase in error. The result of the intersection probability values from 0.15 to 0.4 is linear enough and do not change. But since the value of probability of intersection 0.4 there is an increase of error.

\subsection{Dependence on the number of cells}

The results of processing, depending on the number of cells, are obtained by processing several sets of images grouped by the number of cells in the picture. The results are described by graph in Figure 10.

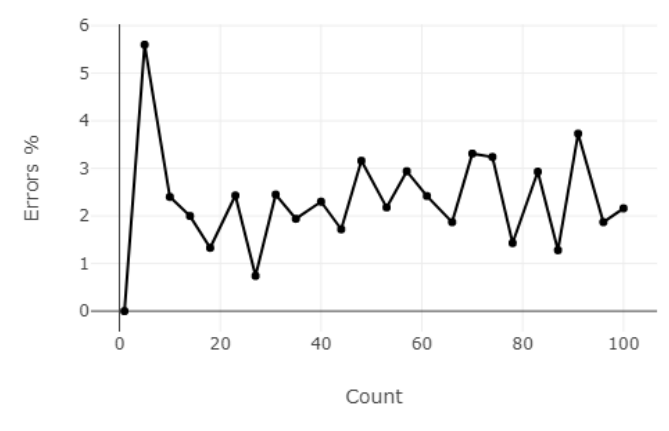

Figure 10. Dependence of number of cells to the error

On the results, there no correlation between the growth of the number of cells and the increase in the amount of error. The ideal work of the algorithm can be seen only on images with one cell. With an increase in the number of cells, the error increases. At most values of the number of cells, an error is between $1 \%$ and $3.5 \%$. 


\subsection{Error estimates}

There are several dominant approaches for analysis, modelling and simulation, Among others are numerical methods, including Finite Difference Time Domain (FDTD), Finite Element Method (FEM) in the frequency domain, Integral Equation (IE) method in the frequency domain. In work [22] the comparison of efficiency for several numerical methods was done, and it was shown that BIE methods, including Spectral Boundary Integral Equation method (SBIE), Method of Auxiliary Sources (MAS) and Multiple Multipole Programm (MMP) are faster and more accurate than those, based on FEM and FD. In this work, the investigation is continued for cell structures. Spectral methods are particularly suited for analysis of such structures, elements of those have shape, similar to such canonical shape, on that particular spectral method is based. Then Spectral Fourier-Galerkin BIE method with analytical regularization based on singularity subtractions in conjunction with conformal mapping approach has been found to be particularly well suited to solve configurable cell segmentation problems, due to the following reasons:

- cells have such cross-section shape, which is close to circular;

- using parameterizations, obtained from conformal mapping approach, allows converting the BIE along polygon to BIE over a circle, and then Fourier harmonics provide natural basis for their discretization;

- integral kernels are regularized analytically using a singularity subtraction technique, leading to smooth integral kernels;

- the latter step also converts all equations to the Fredholm integral equations of the second kind for which a numerical stability is guaranteed;

- it permits an extensive use of FFT in order to obtain a solution with spectral accuracy, reduced complexity and calculation time.

With this method the best possible rate of convergence for solution, i.e. exponential rate of convergence, is achievable. For this purpose, we developed new strategy for accurate error estimation. The main idea of this algorithm is based on splitting of the global error in all local errors, so that it is possible to evaluate all local errors separately. It is particularly suited to solve configurable cell segmentation problem, because many parameters for solving this problem are involved and must be configured. Among others are physical parameters and numerical parameters. To obtain each local error only one numerical parameter must be varied, while all other parameters are kept fixed. Parameters must be fixed in such a way, so that numerical parameters are as big as possible, and physical parameters are those, which present the most complicated case for investigation. Evaluating all local errors, we may choose numerical parameters so that each local error will be as small as possible leading to the best possible global error of whole algorithm. So, obtained solution is those which over performed one, presented in article [19] due to method of error estimate presented in this work and ideas to compare efficiency of several methods presented in [22].

\section{DISCUSSION}

The obtained information about cell structure is used to mimic it, producing nanostructured materials. Since cross-section of the cell has closed boundary, the spectral Fourier-Galerkin method of Boundary Integral Equations with analytical regularization based on the singularity subtraction improved by Fast Fourier Transform (FFT) is most suitable to analyse properties found in natural template, so that it lead to successful nanostructure design. Since particle will be produced from gold, which has dispersion, the shape must be repeated exactly without simplifications and approximations, because even small geometrical detail can cause strong impact of the field enhancement in area of boundary, leading to wrong physical effect. While this method was originally developed to study only elliptical shapes due to known parametrization of ellipse in parametric form, the development of conformal mapping approach to obtain parameterization of arbitrary polygonal shape lead to developing of parametrization 
of arbitrary shape. These achievements appeared useful to study cells, which have smooth closed boundary, reproducible for novel nanostructure design.

On the nanoscale, the shape, size, material properties as well as external surrounding of a particle play a critical role in how it interacts with light. The present work is aimed to provide guidelines for a choice of nanoparticle parameters to be used for new applications in biological sensing and imaging, medical diagnostics, drug development, photo-thermal therapy of cancer and bacterial infection.

Generally the SPRs are identified as peaks in total scattering cross-section (TSCS), that is presented as a function on the excitation wavelength in specified range. In this work we investigate the optical response of plasmonic nanoparticles when $\lambda \in[400 \mathrm{~nm}, 700 \mathrm{~nm}]$. So with specified method we made calculation of TSCS for cell shape of nanoparticle, which will be modified due to our investigation with cell recognition algorithm.

We keep external shape and size of nanoparticle fixed, that is flower shape with $R=50 \mathrm{~nm}(100 \mathrm{~nm}$ from tip to tip). Specified size is chosen so that nanoparticles may be used in medical diagnostics and therapy of cancer as well as inflectional diseases.

Altering the size of internal surface for nanoparticle we investigate the surface plasmon resonances of nanocells (Figure 11). We carried out calculations for seven different solvents, where $n$ been its refractive index, that represent properties of biological liquids.

Comparing the results for different shapes, it is clear that the most sensitive are the resonances corresponding to thin shell in core-shell structure resembling cell shape. When internal size is growing, additional resonances appeared.

\section{CONCLUSION}

Solution of cell segmentation problem with improved accuracy due to performed numerical error analysis allowed application of presented cell segmentation algorithm for implementation in design of plasmonic nanoparticle and their clusters. The shape of cell described with high accuracy as well as aggregation of cells in cluster are needed for design of novel nanostructured devices and artificial materials, properties of those may over perform properties of materials existing in nature. General result of working algorithm has average error of $2.696 \%$ on sample parameters. In addition, this algorithm allows many parameters to be configured, which will allow obtaining an acceptable level of error for a number of data sets of images with different properties. So the data obtained with this algorithm can be used to interpret and analyze cells in the image and the algorithm itself can be improved for a specific data set by configuring parameters. 


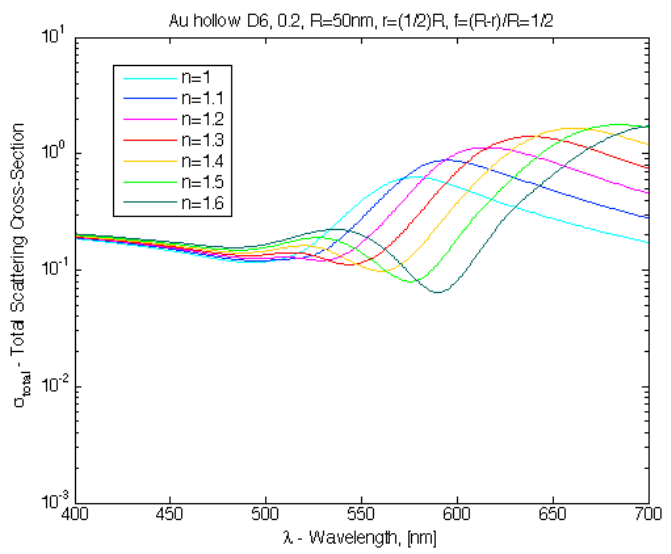

(a)

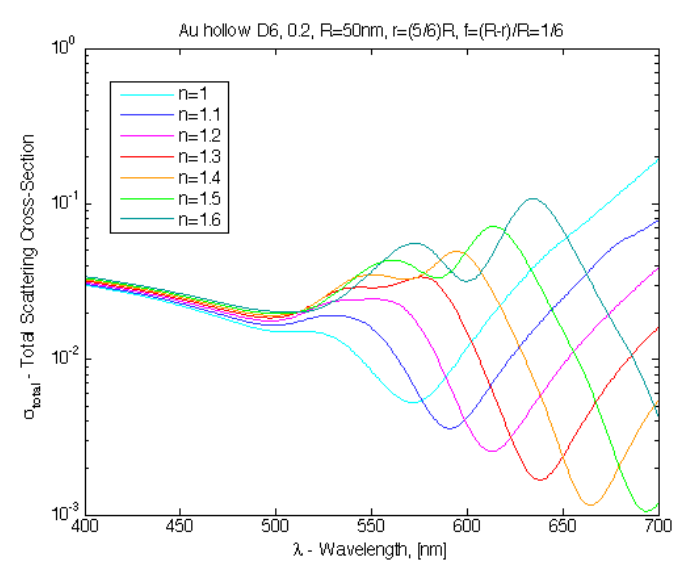

(b)

Figure 11. Scattering cross-sections as functions on an excitation wavelength for gold nanoparticles with $R=50 \mathrm{~nm}(100 \mathrm{~nm}$ from tip to tip), illuminated by an electromagnetic plane wave along $(0,-1)$ direction in various solvents: (a) $r=(1 / 2) R$, (b) $r=(5 / 6) R$.

\section{REFERENCES}

[1] Shim H, Allabergenov B, Kim J, Noh H.Y, Lyu H.-K, Lee M.-J, Choi B. Highly Bright Flexible Electroluminescent Devices with Retroreflective Electrodes, Advanced Materials Technologies 2017; 2(9): 1700040. DOI: 10.1002/admt.201700040

[2] Liapis A.C, Rahman A, Black C.T. Self-assembled nanotextures impart broadband transparency to glass windows and solar cell encapsulants, Appl. Phys. Lett. 2017; 111: 183901. https://doi.org/10.1063/1.500096

[3] Law J. B. K, Ng A.M.H, He A.Y, Low H.Y., Bioinspired Ultrahigh Water Pinning Nanostructures, Langmuir 2014; 30(1): 325-331, DOI: 10.1021/la4034996.

[4] Podsiadlo P, Liu Z, Paterson D, Messersmith P. B, Kotov N.A, Fusion of Seashell Nacre and Marine Bioadhesive Analogs: High-Strength Nanocomposite by Layer-by-Layer Assembly of Clay and L3,4-Dihydroxyphenylalanine Polymer, Advanced Materials 2007; 19(7): 949-955. DOI: 10.1002/adma.200602706.

[5] Davies O.G, Cox S.C, Williams R.L, Tsaroucha D, Dorrepaal R.M, Lewis M.P, Grover L.M, Annexin-enriched osteoblast-derived vesicles act as an extracellular site of mineral nucleation within developing stem cell cultures, Scientific Reports 2017; 7: 12639, doi:10.1038/s41598-01713027-6.

[6] Palkovic S.D, Brommer D.B, Kupwade-Patil K, Masic A., Buehler M.J, Büyüköztürk O, Roadmap across the mesoscale for durable and sustainable cement paste - A bioinspired approach, Construction and Building Materials 2016; 115: 13-31, https://doi.org/10.1016/j.conbuildmat.2016.04.020.

[7] Peng J, Cheng Q, High-Performance Nanocomposites Inspired by Nature, Advanced Materials, 2017; 29: 1702959; DOI: 10.1002/adma.201702959. 
[8] Wong C.K, Mason A.F, Stenzel M.H, Thordarson P, Formation of non-spherical polymersomes driven by hydrophobic directional aromatic perylene interactions, Nature Communications 2017; 8: 1240; DOI:10.1038/s41467-017-01372-z.

[9] Boyko D, Podoroznyuk A, Filatova A. The main stages of image processing in the design of biotechnical systems in medical radiology. The National Technical University - Kharkiv Polytechnic Institute 2012; 85 - 86. (in Russian).

[10] Kovrigin A. Application of the principles of building computer vision systems in the problem of image analysis of cellular structures. Scientific Journal of KubSAU 2007; 1 -3. (in Russian).

[11] Tarkov M. Estimation of the number of cells on the images of cytological plant preparations. A.V. Rzhanov Institute of Semiconductor Physics 2013; 187 - 190. (in Russian).

[12] Romero-Rondón M, Sanabria-Rosas L, Bautista-Rozo L, Mendoza-Castellanos A. Algorithm for detection of overlapped red blood cells in microscopic images of blood smears. DYNA 84; Rzhanov Institute of Semiconductor Physics 2016; 187 - 194.

[13] Uchida S. Image processing and recognition for biological images. Dev. Growth. Differ. 2013; 55(4): 523-549.

[14] Rivest J, Soille P, Baucher S. Morphological gradients. Journal of Electronic Imaging 1993; 2(4).

[15] Krylov V, Shcherbakova G, Pisarenko R, Bilous N. Signal restoration by means of blind deconvolution based on optimization with wavelet transformation In: IEEE: 2016 Third International Scientific-Practical Conference Problems of Infocommunications Science and Technology (PIC S\&T), October 4-6, 2016, Kharkiv, Ukraine; DOI: 10.1109/INFOCOMMST.2016.7905324.

[16] Ablameyko S, Nedzved A. Processing of optical images of cellular structures in medicine. OIIP NAS Belarus 2005; 3: 35-55 (in Russian).

[17] Firdousi R, Parveen S. Local Thresholding Techniques in Image Binarization. International Journal Of Engineering And Computer Science 2014; 3: 4062-4065.

[18] Bradski G, Kaehler A. Learning OpenCV: Computer vision with the OpenCV library. O'Reilly Media San Diego, Inc., 2008.

[19] Hramm O, Bilous N, Ahekian I. Configurable Cell Segmentation Solution Using Hough Circles Transform and Watershed Algorithm.In: 2019 IEEE 8th International Conference on Advanced Optoelectronics and Lasers (CAOL) CAOL 2019 September 6-8, 2019, Sozopol, Bulgaria, USA: IEEE, 602-605.

[20] Image set BBBC005v1 from the Broad Bioimage Benchmark Collection. Available from: https://data.broadinstitute.org/bbbc/BBBC005/

[21] Image set BBBC004v1 from the Broad Bioimage Benchmark Collection. Available from: https://data.broadinstitute.org/bbbc/BBBC004/

[22] Smajic J, Hafner Ch, Raguin L, Tavzarashviki K, Mishrikey M, Comparison of numerical methods for the analysis of plasmonic structures. J. Comput. Theor. Nanoscience 2009; 6: 1-12. 